\title{
Virology, Animal
}

National Cancer Institute

\section{Source}

National Cancer Institute. Virology, Animal. NCI Thesaurus. Code C19113.

The study of animal viruses and the diseases they cause. 